Research Paper

\title{
INR-to-platelet ratio (INPR) as a novel noninvasive index for predicting liver fibrosis in chronic hepatitis $B$
}

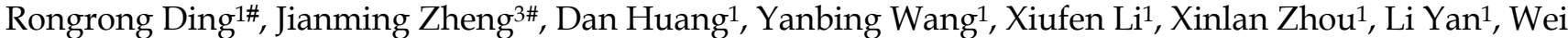 \\ Lu $^{1}$, Zongguo Yang ${ }^{\circledR}$ and Zhanqing Zhang ${ }^{\bowtie}$ \\ 1. Department of Hepatobiliary Medicine, Shanghai Public Health Clinical Center, Fudan University, Shanghai 201508, China \\ 2. Department of Integrative Medicine, Shanghai Public Health Clinical Center, Fudan University, Shanghai 201508, China. \\ 3. Department of Infectious Diseases, Huashan Hospital, Fudan University, Shanghai 200040, China. \\ \#These authors have contributed equally to this work. \\ $\triangle$ Corresponding authors: Zongguo Yang, MD, PhD, Department of Integrative Medicine, Shanghai Public Health Clinical Center, Fudan University, 2901 \\ Caolang Road, Shanghai 201508, China. Tel.: +8621-37990333 ext.3268; E-mail: yangzongguo@shphc.org.cn; ORCID: https://orcid.org/0000-0002-6623-4841; \\ Zhanqing Zhang, MD, Shanghai Public Health Clinical Center, Fudan University, 2901 Caolang Road, Shanghai 201508, China. Tel: +8621-37990333 ext.3245. \\ E-mail: doctorzzqsphc@163.com; ORCID: https:/ / orcid.org/0000-0001-7709-9027.
}

(C) The author(s). This is an open access article distributed under the terms of the Creative Commons Attribution License (https://creativecommons.org/licenses/by/4.0/). See http://ivyspring.com/terms for full terms and conditions.

Received: 2020.08.10; Accepted: 2020.12.18; Published: 2021.01.09

\begin{abstract}
Objective: We aimed to investigate whether a novel noninvasive index, i.e., the international normalized ratio-to-platelet ratio (INPR), was a variable in determining liver fibrosis stage in patients with chronic hepatitis $\mathrm{B}(\mathrm{CHB})$.

Methods: A total of 543 treatment-naïve $\mathrm{CHB}$ patients were retrospectively enrolled. Liver histology was assessed according to the Metavir scoring scheme. All common demographic and clinical parameters were analyzed.

Results: Based on routine clinical parameters (age, sex, $\mathrm{HBeAg}$ status, HBV DNA, hematological parameters, coagulation index, and liver biochemical indicators), a novel index, i.e., the INR-to-platelet ratio (INPR), was developed to magnify the unfavorable effects of liver fibrosis on INR and platelets. The AUCs of INPR for predicting significant fibrosis, advanced fibrosis, and cirrhosis were $0.74,0.76$ and 0.86 , respectively. Compared with APRI, FIB-4, and GPR, the INPR had comparable predictive efficacy for significant fibrosis and better predictive performance for advanced fibrosis and cirrhosis.

Conclusion: INPR could be an accurate, easily calculated and inexpensive index to assess liver fibrosis in patients with $\mathrm{CHB}$. Further studies are needed to verify this indicator and compare it with other noninvasive methods for predicting liver fibrosis in $\mathrm{CHB}$ patients.
\end{abstract}

Key words: international normalized ratio, INR, platelet, liver fibrosis, chronic hepatitis B

\section{Introduction}

Chronic hepatitis B (CHB) is a potentially life-threatening healthcare issue and a major cause of liver cirrhosis and hepatocellular carcinoma $[1,2]$. It is estimated that there are more than 240 million people with $\mathrm{CHB}$ worldwide, and approximately 780,000 people die from complications of hepatitis B every year, including cirrhosis, hepatic failure and hepatocellular carcinoma [1]. Based on early diagnosis and effective antiviral therapy, the prognosis of $\mathrm{CHB}$ can be significantly improved, even if it histologically manifests as advanced fibrosis or cirrhosis [3]. Thus, early staging of hepatic fibrosis and assessing risk in $\mathrm{CHB}$ patients are of great significance.

Liver biopsy is still regarded as the gold standard for the assessment of inflammation and fibrosis. However, it has some limitations, such as invasiveness, sampling variability, and associated risk of complications [4]. Additionally, it is difficult to dynamically follow up on the progression of liver fibrosis. Therefore, several noninvasive methods for evaluating liver fibrosis have been developed to minimize the need for liver biopsies and their 
drawbacks. Among these markers, the aspartate aminotransferase-to-platelet ratio index (APRI) $[5,6]$ and FIB-4 score [7] are the two most widely used methods. Although they have been recommended for the diagnosis of cirrhosis in resource-limited settings by the WHO guidelines [1], their value for assessing CHB patients remains in dispute [5, 8, 9]. GPR has been proposed as a new routine method to identify patients with severe fibrosis or cirrhosis in $\mathrm{CHB}$ patients [10]; however, diagnostic values have varied among studies [11, 12].

Blood platelet count is a simple and inexpensive parameter in routine clinical practice. Previous studies have reported that platelet counts decrease with liver fibrosis progression and have demonstrated their potential predictive performance in liver fibrosis for $\mathrm{CHB}$ patients [13, 14]. Kim HJ et al. [15] reported that the international normalized ratio (INR) was increased in liver cirrhosis. Another study found that INR was correlated independently and significantly with liver fibrosis in chronic hepatitis C patients [16]. Recently, a study by Farid K et al. [17] demonstrated that prothrombin-INR scores contributed to predicting the occurrence of large esophageal varices in patients with hepatitis $C$ virus-induced liver cirrhosis. Therefore, the INR could be considered a potential indicator of liver fibrosis.

Whether combining INR and platelet count can increase the predictive value for liver fibrosis is an attractive issue. In this study, we developed a novel and simple index, the INR-to-platelet ratio (INPR), in $\mathrm{CHB}$ patients and compared the diagnostic value of the INPR with that of the APRI, FIB-4, and GPR.

\section{Materials and Methods}

\section{Ethics Statement}

The study protocol and informed consent documents were reviewed and approved by the Ethics Committee of Shanghai Public Health Clinical Center, Fudan University. All CHB patients provided written informed consent during their admission.

\section{Patients}

Between January 2016 and October 2019, a total of 543 consecutive anti-HBV naïve patients with CHB who had undergone liver biopsies at Shanghai Public Health Clinical Center, Fudan University, were retrospectively enrolled in the study. The inclusion criteria were persistently having serum hepatitis B surface antigens for more than 6 months [18] and a liver biopsy within one week of the blood laboratory examinations. The exclusion criteria were hepatocellular carcinoma, antiviral treatment history, decompensated cirrhosis, inadequate liver biopsy samples $(<1.5 \mathrm{~cm})$, coinfection with other types of viral hepatitis, history of overt alcohol consumption $(>40 \mathrm{~g} / \mathrm{d})$, autoimmune liver disease, hereditary metabolic liver disease, and use of anticoagulant drugs.

\section{Data collection}

Percutaneous liver biopsy was performed using 16-G needle under ultrasound guidance. Liver samples with a minimum length of $1.5 \mathrm{~cm}$ and at least 7 complete portal tracts were fixed in $10 \%$ formalin, embedded in paraffin, and stained with HE, Masson's trichrome and reticulin for histological analysis.

Fasting blood samples were obtained within one week of liver biopsy. Platelets and other blood cells were counted using a Sysmex-XT 4000i automated hematology analyzer. INR and other coagulation indices were measured using a STAR Max automatic coagulation analyzer. ALT, AST and other serum biochemical parameters were measured using an Architectc16000 automatic biochemical analysis system.

The formulas for APRI, FIB-4, and GPR are as follows: $\mathrm{APRI}=(\mathrm{AST}(\mathrm{U} / \mathrm{L}) / \mathrm{ULN}$ of AST $) /$ platelet count $\left(10^{9} / \mathrm{L}\right) \times 100 ;$ FIB-4 $=$ (age $($ years $) \times$ $\mathrm{AST}(\mathrm{U} / \mathrm{L})) /\left(\right.$ platelet count $\left.\left(10^{9} / \mathrm{L}\right) \times(\mathrm{ALT}(\mathrm{U} / \mathrm{L}))^{1 / 2}\right)$; and GPR $=(\mathrm{GGT}(\mathrm{U} / \mathrm{L}) / \mathrm{ULN}$ of GGT $) /$ platelet count $\left(10^{9} / \mathrm{L}\right) \times 100$.

\section{Outcomes}

Liver histology was analyzed by two experienced pathologists who were blinded to other laboratory data according to the Metavir scoring system [19], as follows: F1 (portal fibrosis without septa), F2 (portal fibrosis with rare septa), F3 (numerous fibroses without cirrhosis), and F4 (cirrhosis). In this study, liver fibrosis stages F2-4 were defined as significant fibrosis, F3-4 were defined as advanced fibrosis, and F4 was defined as cirrhosis.

\section{Statistical analysis}

Statistical analysis was performed using IBM SPSS Statistics software, version 26.0 (SPSS Inc., Chicago, IL, USA). Continuous variables are reported as median (interquartile range (IQR)) and were compared by the Kruskal-Wallis test. Categorical variables are reported as proportions and were compared by the chi-square test. Laboratory parameters were classified as binary variables by upper or lower limits of normal. Logistic regression models were used to screen the useful laboratory parameters for assessing liver fibrosis levels. The performances of noninvasive markers for predicting liver fibrosis were evaluated by receiver operating characteristic (ROC) curve analyses using Stata software, version 16.0 (STATA Corp., Texas, USA). A two-sided $\mathrm{P}<0.05$ was considered statistically 
significant.

\section{Results}

\section{Baseline characteristics of CHB patients}

A total of 543 treatment-naïve patients with $\mathrm{CHB}$ were enrolled in the study. The characteristics of all of the recruited patients are summarized in Table 1. The patients were a median age of 37 years old and were mostly male $(67.4 \%)$. Fifty $(9.2 \%)$ patients had metabolic-associated fatty liver disease (MAFLD); 338 $(62.2 \%)$ patients were HBeAg positive. The fibrosis stages were $142(26.2 \%)$ in F1, $147(27.1 \%)$ in F2, 91 $(16.8 \%)$ in F3, and $163(30.0 \%)$ in F4. As the liver fibrosis stage increased, the median levels of ALP, GGT, DBil, globulin, total bile acid, and INR increased, while the median levels of cholinesterase, albumin, prealbumin, and platelet counts decreased (all $P<0.001$ ).

\section{A novel index consisting of INR and platelets for predicting liver fibrosis}

Variables including age, sex, MAFLD, HBsAg, HBeAg, HBV DNA, ALT, AST, ALP, GGT, TBil, DBil, cholinesterase, albumin, globulin, prealbumin, total bile acid, FBG, TC, TG, HDL, LDL, urea, creatinine, eGFR, INR, WBC, RBC, neutrophils, platelets, and hemoglobin were included in the univariate analysis. The presence of significant liver fibrosis (F2-4) was associated with MAFLD, HBV DNA, ALT, AST, ALP, GGT, DBil, prealbumin, cholinesterase, globulin, total bile acid, TC, TG, LDL, urea, INR, and WBC, platelet, hemoglobin, neutrophil, and RBC counts. Multivariable analysis identified ALT, GGT, prealbumin, INR, and platelets as independent predictors of significant liver fibrosis (Table 2). The presence of liver cirrhosis (F4) was associated with age, sex, MAFLD, AST, ALP, GGT, DBil, prealbumin, cholinesterase, total bile acid, TC, LDL, HDL, globulin, INR, and WBC, platelet, hemoglobin, neutrophil, and RBC counts. Multivariable analysis identified albumin, cholinesterase, INR, and platelets as independent predictors of cirrhosis (Table 3). Thus, only INR and platelets were independent predictors of significant fibrosis and cirrhosis (all $\mathrm{P}<0.05$ ).

The associations of INR and platelets with liver histopathology were further analyzed. INR and platelets in relation to the Metavir fibrosis stage are shown in Figure 1a, b. INR was positively correlated with the Metavir score $(\mathrm{r}=0.420, P<0.001)$, while platelets were negatively correlated $(\mathrm{r}=-0.450, P<$ 0.001). Thus, we designed the INR to platelet ratio (INPR) as INR/platelet counts $\left(\times 10^{9} / \mathrm{L}\right) \times 100$ to amplify the difference between the INR and platelets in CHB patients with different stages of fibrosis. INPR was significantly positively correlated with Metavir fibrosis stage with a higher correlation coefficient than GPR, APRI, and FIB-4 ( $\mathrm{r}=0.494,0.489,0.453$, and 0.428 , respectively) (Figure $\mathbf{1} \mathbf{c}-\mathbf{f}$ ).

\section{Comparison of INPR, APRI, FIB-4, and GPR in predicting significant liver fibrosis, advanced fibrosis, and cirrhosis in patients with CHB}

The study calculated the data comparing the fourth serum fibrosis scores related to significant liver fibrosis, advanced fibrosis, and cirrhosis in patients with $\mathrm{CHB}$ by ROC curve analysis. The ROC curves of INPR, APRI, FIB-4, and GPR are shown in Table 4. In determining significant liver fibrosis (F2-4), the AUROCs of INPR, APRI, FIB-4, and GPR were 0.74 (sensitivity $57.62 \%$, specificity $80.34 \%$ ), 0.77 (sensitivity $71.15 \%$, specificity $72.63 \%$ ), 0.72 (sensitivity $56.59 \%$, specificity $78.21 \%$ ), and 0.75 (sensitivity $74.45 \%$, specificity $68.72 \%$ ), respectively. When distinguishing advanced liver fibrosis (F3-4), the AUROCs of INPR, APRI, FIB-4, and GPR were 0.76 (sensitivity $67.06 \%$, specificity $75.61 \%$ ), 0.70 (sensitivity 73.23\%, specificity 759.52\%), 0.71 (sensitivity $59.84 \%$, specificity $73.36 \%$ ), and 0.74 (sensitivity $74.80 \%$, specificity $566.44 \%$ ), respectively. For discriminating cirrhosis (F4), the AUROCs of INPR, APRI, FIB-4, and GPR were 0.86 (sensitivity $68.75 \%$, specificity $87.07 \%$ ), 0.74 (sensitivity $83.23 \%$, specificity $55.76 \%$ ), 0.80 (sensitivity $60.87 \%$, specificity $86.13 \%$ ), and 0.80 (sensitivity $75.16 \%$, specificity $76.70 \%)$, respectively. Comparing ROC curves using the DeLong method, the INPR showed comparable performance to GPR, APRI, and FIB-4 for assessing significant fibrosis but significantly better performance for predicting cirrhosis than the other three biomarker panels (all $P<0.05$, Figure 2).

\section{Discussion}

Timely assessment of liver fibrosis stage is extremely important in patients with CHB [20]. Early diagnosis and continuous follow-up of liver fibrosis progression are essential to prevent liver cirrhosis and end-stage liver disease. Liver biopsy is still considered the gold standard method to diagnose liver fibrosis stage, but some drawbacks significantly limit its clinical application [21]. Therefore, many noninvasive methods and model designs with advantages of convenience and good repetition have been developed to stage liver fibrosis in recent years. Transient elastography (TE), shear wave elastography (SWE), and magnetic resonance elastography (MRE) have been reported to have good performances in predicting liver fibrosis stage [22-24]. However, they are expensive, and available equipment is lacking in resource-limited areas. Serum markers such as APRI, 
FIB-4 and GPR have been applied to noninvasively predict liver fibrosis stage [25-27]. However, the sensitivity and specificity of diagnosis by these markers have been limited [28].

In this retrospective study, we developed a simple and inexpensive marker consisting of INR and platelet count to predict liver fibrosis in patients with CHB. Prothrombin time-INR increases along with the progression of liver fibrosis, while platelet counts decrease, and this abnormal condition is especially obvious in patients with cirrhosis $[29,30]$. Liang et al. [31] reported that INR and platelet counts were independent predictors of cirrhosis in patients with CHB. In the study by Kayadibi et al. [32], the AUROC of platelet count for determining significant liver fibrosis was 0.827 in patients with chronic HCV. Thrombocytopenia in liver disease is due to the accumulation and destruction of platelets in portal
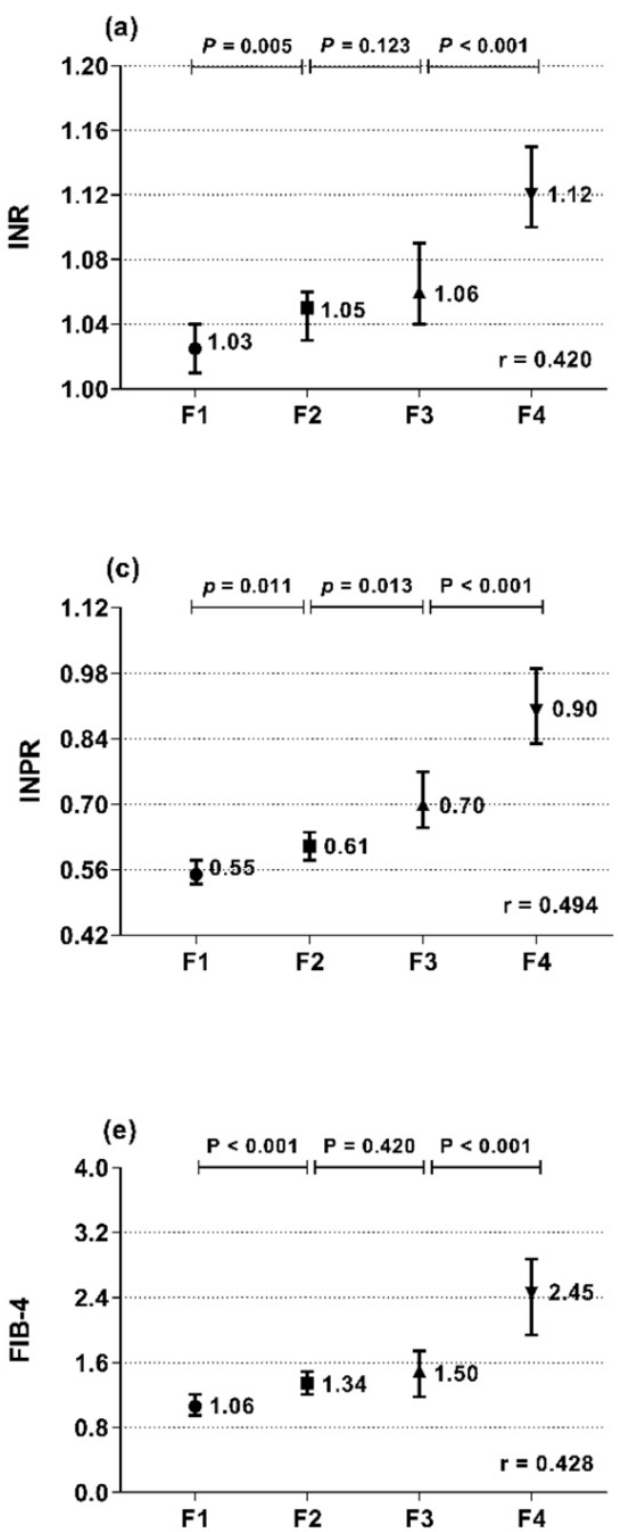

hypertensive splenomegaly, arising from progressive liver fibrosis and partly due to impaired production of thrombopoietin in cirrhosis [33, 34]. In the present study, the INPR significantly increased as the liver fibrosis stage increased, while hepatic function, such as cholinesterase, albumin, and prealbumin, significantly decreased as liver fibrosis progressed. By ROC analysis, the INPR showed good performance for staging significant fibrosis (F2-4), with an AUROC of 0.74. Notably, the performance was excellent for predicting advanced fibrosis (F3-4) and cirrhosis (F4), with AUROCs of 0.76 and 0.86, respectively. In comparison with APRI, FIB-4, and GPR, the performance of the INPR showed larger AUROCs for diagnoses of both F3-4 and F4, suggesting that it sufficiently reflected the amount of accumulated fibrosis tissue in the liver.
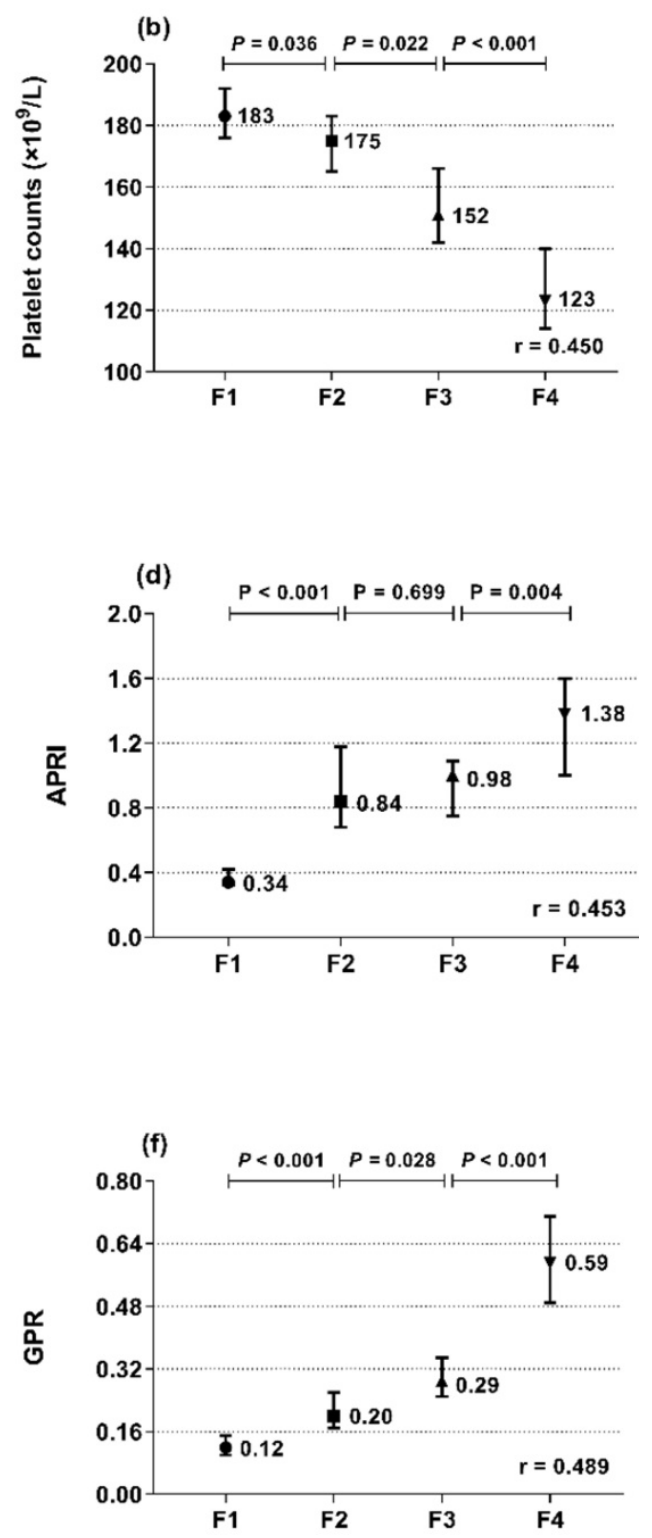
Figure 1. Median and 95\% Cl of INR (a), platelet count (b), INPR (c), APRI (d), FIB-4 (e), and GPR (f) in the four subgroups (F1, F2, F3, and F4) classified by fibrosis stage (Metavir scores). $r$ is the correlation coefficient of the variables with the fibrosis stages.

Table 1. Baseline characteristics of enrolled patients with chronic hepatitis B

\begin{tabular}{|c|c|c|c|c|c|c|}
\hline Variables & Total $(n=543)$ & $\mathrm{F} 1(\mathrm{n}=142)$ & $\mathrm{F} 2(\mathrm{n}=147)$ & $\mathrm{F} 3(\mathrm{n}=91)$ & $\mathrm{F} 4(\mathrm{n}=163)$ & $P$ value \\
\hline Age, years & $37(31-46)$ & $39(30-49)$ & $36(30-43)$ & $36(31-46)$ & $39(32-47)$ & 0.145 \\
\hline Male, n (\%) & $366(67.4)$ & $96(67.6)$ & $93(63.3)$ & $63(69.2)$ & $114(69.9)$ & 0.333 \\
\hline MAFLD, n (\%) & $50(9.2)$ & 25 (17.6) & $10(6.8)$ & $6(6.6)$ & $9(5.5)$ & 0.001 \\
\hline $\log _{10}[\mathrm{HBsAg}], \mathrm{IU} / \mathrm{ml}$ & $3.55(3.21-4.10)$ & $3.55(3.06-4.12)$ & $3.86(3.41-4.35)$ & $3.52(3.11-3.95)$ & $3.45(3.09-3.72)$ & $<0.001$ \\
\hline HBeAg positive, $\mathrm{n}(\%)$ & $338(62.2)$ & $76(53.5)$ & $106(72.1)$ & $62(68.1)$ & $94(57.7)$ & 0.004 \\
\hline $\log _{10}[\mathrm{HBVDNA}], \mathrm{IU} / \mathrm{ml}$ & $6.33(4.19-7.310$ & $4.67(2.01-6.70)$ & $7.02(4.96-7.67)$ & $6.28(4.74-7.21)$ & $6.25(4.54-7.10)$ & $<0.001$ \\
\hline ALT, U/L & $64.00(32.00-157.00)$ & $34.00(18.75-72.75)$ & $94.00(43.00-183.00)$ & $79.00(39.00-211.00)$ & $67.00(38.00-177.00)$ & $<0.001$ \\
\hline AST, U/L & $46.00(27.00-95.00)$ & $27.00(19.00-40.50)$ & $53.00(31.00-113.00)$ & $53.00(31.00-102.00)$ & $59.00(35.00-117.00)$ & $<0.001$ \\
\hline $\mathrm{ALP}, \mathrm{U} / \mathrm{L}$ & $80.00(65.00-101.00)$ & $71.00(59.00-85.00)$ & $77.00(63.00-97.00)$ & $81.00(66.00-97.00)$ & $97.00(76.00-119.00)$ & $<0.001$ \\
\hline GGT, U/L & $41.00(21.00-85.00)$ & $21.00(15.00-45.25)$ & $35.00(18.00-89.00)$ & $45.00(26.00-85.00)$ & $67.00(37.00-123.00)$ & $<0.001$ \\
\hline TBil, $\mu \mathrm{mol} / \mathrm{L}$ & $15.60(11.40-21.80)$ & $14.60(11.30-18.00)$ & $14.40(10.60-19.40)$ & $14.70(11.10-19.80)$ & $18.20(13.00-28.00)$ & $<0.001$ \\
\hline $\mathrm{DBil} \mu \mathrm{mol} / \mathrm{L}$ & $5.80(4.30-8.40)$ & $5.20(4.00-6.43)$ & $5.50(4.00-7.40)$ & $5.60(4.30-8.20)$ & $7.50(5.20-15.10)$ & $<0.001$ \\
\hline Cholinesterase, $\mathrm{U} / \mathrm{L}$ & $7391.00(6002.00-8787.00)$ & $9072.00(7520.00-10493.50)$ & $7505.00(6501.50-8550.50)$ & $7361.50(6344.00-8323.25)$ & $5994.00(4491.00-7328.00)$ & $<0.001$ \\
\hline Albumin, g/L & $41.70(38.60-44.40)$ & $43.05(41.00-46.00)$ & $42.00(38.90-44.40)$ & $41.20(39.10-44.60)$ & $39.00(35.10-43.00)$ & $<0.001$ \\
\hline Globulin, $\mathrm{g} / \mathrm{L}$ & $30.00(26.83-33.00)$ & $29.00(25.00-31.00)$ & $30.00(27.00-32.00)$ & $29.00(27.00-32.00)$ & $31.00(27.00-35.00)$ & $<0.001$ \\
\hline Prealbumin, g/L & $185.00(131.00-241.88)$ & $249.70(204.75-291.65)$ & $177.00(131.00-221.63)$ & $174.00(138.50-215.08)$ & $148.00(100.00-203.64)$ & $<0.001$ \\
\hline Total bile acid, $\mu \mathrm{mol} / \mathrm{L}$ & $9.70(4.60-18.95)$ & $4.90(2.80-9.13)$ & $9.10(4.65-17.55)$ & $10.40(5.45-15.70)$ & $17.90(9.58-45.38)$ & $<0.001$ \\
\hline $\mathrm{FBG}, \mathrm{mmol} / \mathrm{L}$ & $4.79(4.47-5.20)$ & $4.91(4.65-5.28)$ & $4.77(4.46-5.20)$ & $4.76(4.49-5.13)$ & $4.71(4.34-5.13)$ & 0.007 \\
\hline $\mathrm{TC}, \mathrm{mmol} / \mathrm{L}$ & $4.14(3.65-4.81)$ & $4.32(3.87-5.05)$ & $4.20(3.71-4.85)$ & $4.10(3.61-4.87)$ & $3.87(3.42-4.56)$ & $<0.001$ \\
\hline $\mathrm{TG}, \mathrm{mmol} / \mathrm{L}$ & $0.94(0.72-1.28)$ & $1.00(0.81-1.50)$ & $0.91(0.70-1.24)$ & $0.91(0.71-1.22)$ & $0.93(0.71-1.26)$ & 0.023 \\
\hline $\mathrm{HDL}, \mathrm{mmol} / \mathrm{L}$ & $1.31(1.03-1.62)$ & $1.27(1.02-1.57)$ & $1.39(1.12-1.67)$ & $1.36(1.09-1.68)$ & $1.19(0.95-1.63)$ & 0.001 \\
\hline LDL mmol/L & $2.59(2.10-3.17)$ & $2.75(2.29-3.48)$ & $2.59(2.19-3.21)$ & $2.55(2.07-3.03)$ & $2.39(1.99-2.90)$ & $<0.001$ \\
\hline Urea, mmol/L & $310.25(253.00-365.40)$ & $328.00(262.00-382.00)$ & $293.00(243.10-369.10)$ & $322.00(262.75-364.25)$ & $303.50(252.75-341.62)$ & 0.032 \\
\hline Creatinine, $\mu \mathrm{mol} / \mathrm{L}$ & $65.20(54.95-74.70)$ & $64.10(56.60-74.15)$ & $63.40(54.08-74.90)$ & $67.75(54.90-73.78)$ & $65.70(54.50-75.85)$ & 0.819 \\
\hline $\mathrm{eGFR}, \mathrm{ml} /\left(\min \times 1.73 \mathrm{~m}^{2}\right)$ & $116.42(102.71-133.15)$ & $115.55(101.90-133.70)$ & $120.55(102.82-135.01)$ & $112.42(102.75-130.67)$ & $116.57(102.89-133.42)$ & 0.537 \\
\hline INR & $1.06(1.01-1.13)$ & $1.03(0.98-1.07)$ & $1.05(1.00-1.10)$ & $1.06(1.01-1.14)$ & $1.12(1.06-1.22)$ & $<0.001$ \\
\hline WBC count, $\times 10^{9} / \mathrm{L}$ & $5.24(4.22-6.22)$ & $5.58(4.64-6.63)$ & $5.09(4.34-6.01)$ & $5.13(4.22-6.12)$ & $4.85(3.91-6.11)$ & 0.003 \\
\hline RBC count, $\times 10^{9} / \mathrm{L}$ & $4.61(4.23-4.98)$ & $4.81(4.44-5.10)$ & $4.60(4.29-4.98)$ & $4.55(4.26-4.95)$ & $4.48(3.97-4.91)$ & $<0.001$ \\
\hline Platelet count, $\times 10^{9} / \mathrm{L}$ & $159.00(126.00-196.00)$ & $183.00(156.75-216.00)$ & $175.00(147.00-201.00)$ & $152.00(126.00-192.00)$ & $123.00(89.00-157.00)$ & $<0.001$ \\
\hline Neutrophils count, $\times 10^{9} / \mathrm{L}$ & $2.81(2.16-3.54)$ & $3.21(2.55-3.96)$ & $2.72(2.26-3.42)$ & $2.64(2.11-3.49)$ & $2.53(1.93-3.30)$ & $<0.001$ \\
\hline Hemoglobin, g/L & $145.00(130.00-155.00)$ & $147.50(133.75-157.00)$ & $145.00(131.00-156.00)$ & $146.00(131.00-155.00)$ & $140.00(125.00-154.00)$ & 0.036 \\
\hline
\end{tabular}

MAFLD, metabolic associated fatty liver disease; FBG, fasting blood glucose; TC, total cholesterol; TG, total triglycerides; HDL, high density lipoprotein; LDL, low density

lipoprotein; eGFR, estimated glomerular filtration rate; WBC, white blood cell; RBC, red blood cell.

Table 2. Univariate and multivariate analyses of clinical items and significant fibrosis in patients with $\mathrm{CHB}(\mathrm{n}=364)$

\begin{tabular}{|c|c|c|c|c|}
\hline \multirow[t]{3}{*}{ Variables } & \multicolumn{4}{|c|}{ Significant fibrosis (F2-4) } \\
\hline & \multicolumn{2}{|c|}{ Univariate } & \multicolumn{2}{|l|}{ Multivariate } \\
\hline & OR $(95 \% \mathrm{CI})$ & $P$ value & OR $(95 \%$ CI) & $P$ value \\
\hline MAFLD, yes vs. no & $0.32(0.18-0.57)$ & $<0.001$ & ---- & ---- \\
\hline $\log _{10}[\mathrm{HBVDNA}], \mathrm{IU} / \mathrm{ml}$ & $1.32(1.19-1.46)$ & $<0.001$ & --- & --- \\
\hline $\mathrm{ALT}, \mathrm{U} / \mathrm{L}$ & $1.00(1.00-1.01)$ & $<0.001$ & $0.99(0.99-0.99)$ & 0.002 \\
\hline AST, U/L & $1.01(1.00-1.01)$ & $<0.001$ & --- & - \\
\hline $\mathrm{ALP}, \mathrm{U} / \mathrm{L}$ & $1.01(1.01-1.02)$ & $<0.001$ & ---- & --- \\
\hline GGT, U/L & $1.01(1.00,1.01)$ & $<0.001$ & $1.02(1.01-1.04)$ & $<0.001$ \\
\hline DBil, umol/L & 1.09 (1.04-1.14) & $<0.001$ & --- & ---- \\
\hline Prealbumin, g/L & $0.98(0.98-0.99)$ & $<0.001$ & $0.99(0.98-0.99)$ & $<0.001$ \\
\hline Cholinesterase, U/L & $1.00(0.99-1.00)$ & $<0.001$ & --- & ---- \\
\hline Globulin, g/L & $1.08(1.03-1.12)$ & $<0.001$ & ---- & --- \\
\hline Total bile acid, $\mu \mathrm{mol} / \mathrm{L}$ & $1.04(1.02-1.06)$ & $<0.001$ & ---- & --- \\
\hline $\mathrm{TC}, \mathrm{mmol} / \mathrm{L}$ & $0.65(0.52-0.80)$ & $<0.001$ & --- & --- \\
\hline $\mathrm{TG}, \mathrm{mmol} / \mathrm{L}$ & $0.53(0.38-0.76)$ & $<0.001$ & ---- & --- \\
\hline $\mathrm{LDL}, \mathrm{mmol} / \mathrm{L}$ & $0.57(0.45-0.72)$ & $<0.001$ & --- & --- \\
\hline Urea, mmol/L & $0.99(0.99-1.00)$ & 0.040 & --- & ---- \\
\hline INR & $2.57(1.99-3.32)$ & $<0.001$ & $1.75(1.20-2.59)$ & 0.005 \\
\hline WBC count, $\times 10^{9} / \mathrm{L}$ & $0.79(0.71-0.90)$ & $<0.001$ & 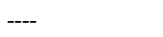 & ---- \\
\hline Platelet count, $\times 10^{9} / \mathrm{L}$ & $0.99(0.98-0.99)$ & $<0.001$ & $0.99(0.98-0.99)$ & 0.005 \\
\hline Hemoglobin, g/L & $0.98(0.97-0.99)$ & 0.015 & --- & ---- \\
\hline Neutrophil count, $\times 10^{9} / \mathrm{L}$ & $0.61(0.51-0.73)$ & $<0.001$ & --- & --- \\
\hline RBC count, $\times 10^{12} / \mathrm{L}$ & $0.44(0.31-0.62)$ & $<0.001$ & ---- & --- \\
\hline
\end{tabular}

Variables including age, sex, MAFLD, HBsAg quantity, HBeAg status, HBVDNA, ALT, AST, ALP, GGT, TBil, DBil, prealbumin, albumin, cholinesterase, total bile acid, FBG, TC, TG, LDL, HDL, urea, creatinine, eGFR, INR, WBC, RBC, platelet, neutrophil, and hemoglobin were included in the univariate model.

Only variables significantly associated with significant fibrosis in univariate analysis were presented and enrolled in multivariate model.
Table 3. Univariate and multivariate analyses of clinical items and cirrhosis in patients with $\mathrm{CHB}(\mathrm{n}=161)$

\begin{tabular}{|c|c|c|c|c|}
\hline \multirow[t]{3}{*}{ Variables } & \multicolumn{4}{|l|}{ Cirrhosis (F4) } \\
\hline & \multicolumn{2}{|l|}{ Univariate } & \multicolumn{2}{|l|}{ Multivariate } \\
\hline & OR $(95 \% \mathrm{CI})$ & $P$ value & OR $(95 \%$ CI $)$ & $P$ value \\
\hline Age, years & $1.02(1.00-1.04)$ & 0.014 & ---- & ---- \\
\hline Gender, male & $1.55(1.03-2.33)$ & 0.036 & ---- & ---- \\
\hline MAFLD, yes vs. no & $0.36(0.16-0.82)$ & 0.014 & ---- & ---- \\
\hline AST, U/L & $1.00(1.00-1.00)$ & 0.001 & ---- & ---- \\
\hline $\mathrm{ALP}, \mathrm{U} / \mathrm{L}$ & $1.01(1.00-1.02)$ & $<0.001$ & ---- & ---- \\
\hline GGT, U/L & $1.00(1.00-1.01)$ & 0.001 & ---- & ---- \\
\hline DBil, $\mu \mathrm{mol} / \mathrm{L}$ & 1.05 (1.03-1.07) & $<0.001$ & ---- & ---- \\
\hline Prealbumin, g/L & $0.99(0.99-1.00)$ & $<0.001$ & ---- & --- \\
\hline Albumin, g/L & $0.84(0.80-0.88)$ & $<0.001$ & $0.89(0.83-0.97)$ & 0.006 \\
\hline Cholinesterase, U/L & $0.99(0.99,1.00)$ & $<0.001$ & $1.00(0.99-1.00)$ & 0.001 \\
\hline $\begin{array}{l}\text { Total bile acid, } \\
\mu \mathrm{mol} / \mathrm{L}\end{array}$ & $1.02(1.01,1.03)$ & $<0.001$ & ---- & ---- \\
\hline $\mathrm{TC}, \mathrm{mmol} / \mathrm{L}$ & $0.57(0.45-0.73)$ & $<0.001$ & ---- & ---- \\
\hline $\mathrm{LDL}, \mathrm{mmol} / \mathrm{L}$ & $0.59(0.45-0.77)$ & $<0.001$ & --- & --- \\
\hline $\mathrm{HDL}, \mathrm{mmol} / \mathrm{L}$ & $0.45(0.27-0.73)$ & 0.001 & --- & -- \\
\hline Globulin, g/L & 1.08 (1.04-1.12) & $<0.001$ & --- = & --- \\
\hline INR & $3.15(2.46-4.04)$ & $<0.001$ & 2.52 (1.72-3.68) & $<0.001$ \\
\hline WBC count, $\times 10^{9} / \mathrm{L}$ & $0.78(0.69-0.90)$ & $<0.001$ & - & --- \\
\hline $\begin{array}{l}\text { Platelet count, } \\
\times 10^{9} / \mathrm{L}\end{array}$ & $0.97(0.96-0.97)$ & $<0.001$ & $0.97(0.96-0.98)$ & $<0.001$ \\
\hline Hemoglobin, g/L & $0.99(0.98-1.00)$ & 0.045 & --- & ---- \\
\hline $\begin{array}{l}\text { Neutrophil count, } \\
\times 10^{9} / \mathrm{L}\end{array}$ & $0.69(0.57-0.84)$ & $<0.001$ & ---- & ---- \\
\hline RBC count, $\times 10^{12} / \mathrm{L}$ & $0.47(0.33-0.65)$ & $<0.001$ & ---- & --- \\
\hline
\end{tabular}

Variables including age, sex, MAFLD, HBsAg quantity, HBeAg status, HBVDNA, ALT, AST, ALP, GGT, TBil, DBil, prealbumin, albumin, cholinesterase, total bile 
A

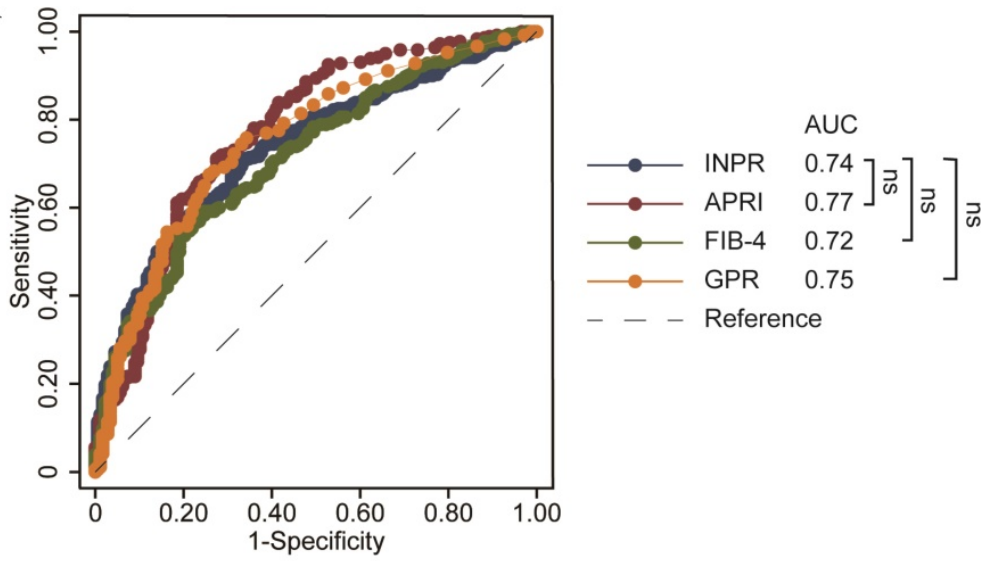

B

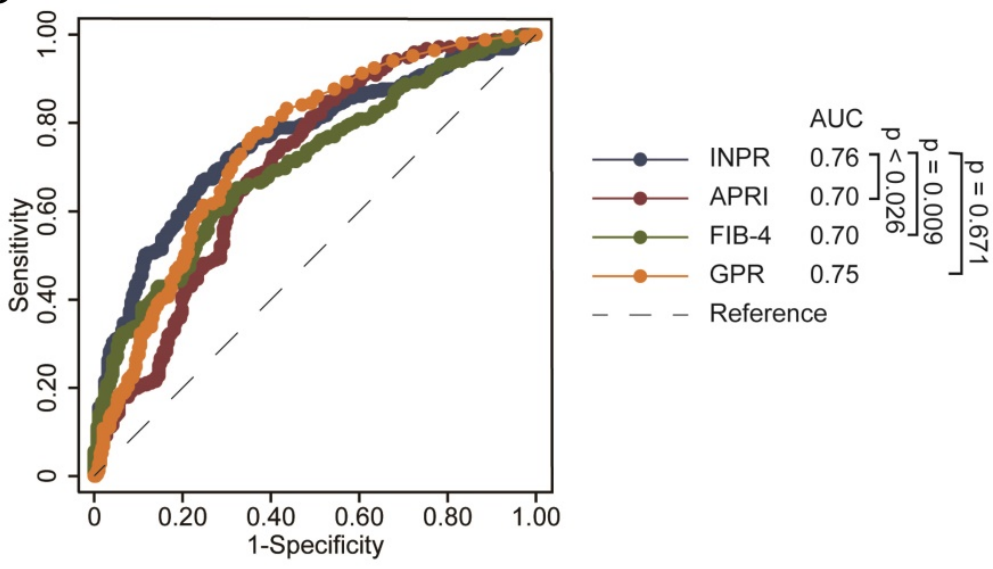

C

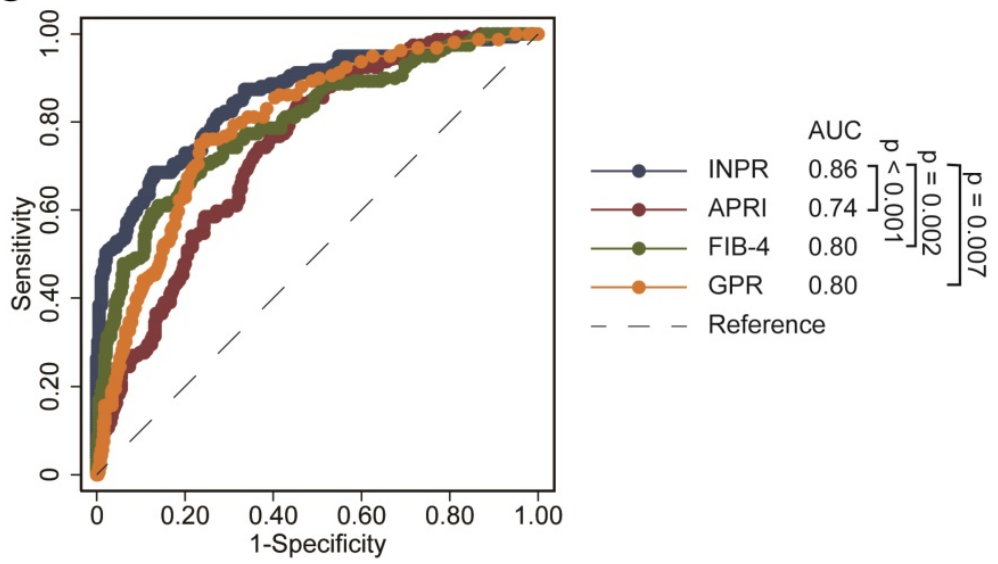

Figure 2. ROC comparison of INPR, APRI, FIB-4, and GPR for predicting significant fibrosis and cirrhosis. (a) ROC comparison for predicting significant fibrosis; (b) ROC comparison for predicting advanced fibrosis; (c) ROC comparison for predicting cirrhosis.

acid, FBG, TC, TG, LDL, HDL, urea, creatinine, eGFR, INR, WBC, RBC, platelet, neutrophil, and hemoglobin were included in the univariate model.

Only variables significantly associated with cirrhosis in univariate analysis were presented and enrolled in multivariate model.

APRI and FIB-4 are the two most widely studied noninvasive serum models, and they have been recommended for detecting liver fibrosis and cirrhosis in resource-limited settings by the WHO HBV guidelines due to their very low cost, easy access, and regular use testing order in clinical practice, and they have been substantially used to identify liver fibrosis and cirrhosis [1]. Although previous studies have demonstrated that APRI and FIB-4 had good performance for the identification of significant fibrosis and cirrhosis [35, 36], their accuracy is disputed in patients with CHB $[25,26]$. Some studies have shown that APRI and FIB-4 evaluated liver fibrosis in CHB patients with moderate accuracy, but they might not be ideal measures to predict liver fibrosis [6, 37]. In our study, APRI exhibited AUROCs of 0.77 for predicting significant fibrosis and 0.74 for detecting cirrhosis, similar to previous studies [38-40]. The performance of FIB-4 was relatively moderate for the prediction of F2-4, with an AUROC of 0.72. In line with the results from previous studies, FIB-4 showed good performance for detecting cirrhosis, with an AUROC of 0.80 [38].

GPR is a new noninvasive marker for liver fibrosis in patients with chronic HBV infection despite the diagnostic value of GPR being atypical [10, 11, 41-43]. Our findings demonstrated the good performance of GPR in predicting significant fibrosis, advanced fibrosis and cirrhosis, with AUROCs of 0.75, 0.74, and 0.80 . This outcome matched with a previous study that showed that GPR was a promising predictor of significant fibrosis and cirrhosis [10, 42]. Recent studies [44, 45] have analyzed the diagnostic accuracy of GPR according to HBeAg status. In the studies by Dong et al. [44] and Peng et al. [45] for HBeAg-positive CHB, GPR was better than APRI in predicting advanced fibrosis and cirrhosis but was comparable to FIB-4 in distinguishing significant fibrosis, advanced fibrosis and cirrhosis; however, for $\mathrm{HBeAg}$-negative $\mathrm{CHB}$, the predictive performance of GPR in assessing significant fibrosis, advanced fibrosis and cirrhosis was comparable to that of FIB-4 and APRI. Therefore, HBeAg status is not the main factor leading to the difference. We believe that differences in basic characteristics, sample size, spectrum bias of fibrosis distribution, HBV genotypes and different histological scoring systems will lead to differences in results.

By optimized cutoff values of the INPR, significant fibrosis, advanced fibrosis, and cirrhosis could be accurately diagnosed in $64.9 \%, 71.4 \%$ and $81.3 \%$ of $\mathrm{CHB}$ patients, respectively. The diagnostic 
accuracies of APRI, FIB-4, and GPR for advanced fibrosis were $65.5 \%, 66.6 \%$, and $69.6 \%$, respectively. The diagnostic accuracies of APRI, FIB-4, and GPR for cirrhosis were $67.4 \%, 78.4 \%$, and $76.1 \%$, respectively. The current results indicated that the INPR showed better diagnostic accuracy than the other three noninvasive markers in predicting advanced fibrosis and cirrhosis.

Table 4. Predictive values of INPR, APRI, FIB-4 and GPR for liver fibrosis in $\mathrm{CHB}$ patients

\begin{tabular}{|c|c|c|c|c|c|c|}
\hline & \multicolumn{2}{|c|}{$\mathrm{F} 2-4(\mathrm{n}=364)$} & \multicolumn{2}{|c|}{ F3-4 $(\mathrm{n}=254)$} & \multicolumn{2}{|c|}{$\mathrm{F} 4(\mathrm{n}=161)$} \\
\hline & Estimate & $95 \% \mathrm{CI}$ & Estimate & $95 \% \mathrm{CI}$ & Estimate & $95 \% \mathrm{CI}$ \\
\hline \multicolumn{7}{|l|}{ INPR } \\
\hline AUROC & 0.74 & $0.70-0.78$ & 0.76 & $0.72-0.79$ & 0.86 & $0.83-0.90$ \\
\hline Cutoff & 0.69 & - & 0.72 & & 0.83 & - \\
\hline Sen $(\%)$ & 57.62 & $52.3-62.8$ & 67.06 & $60.9-72.9$ & 68.75 & $61.0-75.8$ \\
\hline Spe (\%) & 80.34 & $73.7-85.9$ & 75.61 & $70.2-80.5$ & 87.07 & $83.3-90.3$ \\
\hline PPV (\%) & 85.6 & $81.3-89.0$ & 70.7 & 65.9-75.1 & 69.2 & $62.9-74.8$ \\
\hline NPV (\%) & 48.3 & $44.8-51.8$ & 72.3 & $68.4-75.9$ & 86.8 & 83.9-89.3 \\
\hline DA (\%) & 64.9 & & 71.4 & & 81.3 & \\
\hline \multicolumn{7}{|l|}{ APRI } \\
\hline AUROC & 0.77 & $0.73-0.80$ & 0.70 & $0.66-0.74$ & 0.74 & $0.71-0.78$ \\
\hline Cutoff & 0.66 & - & 0.69 & & 0.69 & - \\
\hline Sen $(\%)$ & 71.15 & $66.2-75.8$ & 73.23 & 67.3-78.6 & 83.23 & $76.5-88.6$ \\
\hline Spe (\%) & 72.63 & $65.5-79.0$ & 59.52 & $53.6-65.2$ & 55.76 & $50.6-60.8$ \\
\hline PPV (\%) & 84.10 & $80.5-87.1$ & 61.4 & $57.6-65.1$ & 44.2 & $41.0-47.5$ \\
\hline NPV (\%) & 55.30 & $50.7-59.8$ & 71.7 & $66.9-76.0$ & 88.7 & 84.7-91.8 \\
\hline DA (\%) & 71.6 & & 65.5 & & 67.4 & \\
\hline \multicolumn{7}{|l|}{ FIB-4 } \\
\hline AUROC & 0.72 & $0.68-0.75$ & 0.71 & $0.67-0.74$ & 0.80 & $0.76-0.83$ \\
\hline Cutoff & 1.56 & - & 1.65 & & 2.33 & - \\
\hline Sen $(\%)$ & 56.59 & 51.3-61.8 & 59.84 & $53.5-65.9$ & 60.87 & $52.9-68.5$ \\
\hline Spe (\%) & 78.21 & $71.4-84.0$ & 73.36 & 67.9-78.4 & 86.13 & $82.2-89.4$ \\
\hline PPV (\%) & 84.1 & 79.8-87.6 & 66.4 & 61.4-71.0 & 64.9 & $58.3-71.0$ \\
\hline NPV (\%) & 47.0 & $43.5-50.5$ & 67.5 & $63.8-71.0$ & 83.9 & $81.1-86.4$ \\
\hline DA (\%) & 63.3 & & 66.6 & & 78.4 & \\
\hline \multicolumn{7}{|l|}{ GPR } \\
\hline AUROC & 0.75 & $0.70-0.78$ & 0.74 & $0.71-0.78$ & 0.80 & $0.76-0.83$ \\
\hline Cutoff & 0.20 & & 0.25 & & 0.38 & \\
\hline Sen $(\%)$ & 74.45 & $69.6-78.9$ & 74.80 & $69.0-80.0$ & 75.16 & $67.7-81.6$ \\
\hline Spe (\%) & 68.72 & $61.4-75.4$ & 66.44 & $60.7-71.9$ & 76.70 & $72.1-80.9$ \\
\hline PPV (\%) & 82.9 & 79.4-85.8 & 66.2 & $62.1-70.0$ & 57.6 & $52.6-62.5$ \\
\hline NPV (\%) & 56.9 & $52.0-61.8$ & 75.0 & $70.5-79.0$ & 88.0 & $84.8-90.6$ \\
\hline DA (\%) & 72.0 & & 69.6 & & 76.1 & \\
\hline
\end{tabular}

Sen, sensitivity; Spe, specificity; PPV, positive predictive value; NPV, negative predictive value; DA, diagnostic accuracy.

This study had some limitations. First, it was a retrospective study, which might essentially have incurred selective bias. Thus, the diagnostic performance of INPR must be confirmed in prospective studies. Second, we cannot compare the performance of FibroScan to the INPR because of the lack of TE data for most enrolled patients.

In conclusion, our study indicated that the INPR could be a potentially useful, easily calculated and inexpensive index to predict liver fibrosis in patients with $\mathrm{CHB}$, especially in resource-limited settings. Further studies are needed to verify this indicator and compare it with other noninvasive methods for predicting liver fibrosis in $\mathrm{CHB}$ patients.

\section{Acknowledgements}

This work was supported by the Medical Guidance Project of Shanghai Science and Technology Committee (19401931600). The funders played no role in the study design, data collection and analysis, decision to publish, or preparation of the manuscript.

\section{Competing Interests}

The authors have declared that no competing interest exists.

\section{References}

1. In: Guidelines for the Prevention, Care and Treatment of Persons with Chronic Hepatitis B Infection. Geneva. 2015.

2. Terrault NA, Bzowej NH, Chang KM, Hwang JP, Jonas MM, Murad MH, et al. AASLD guidelines for treatment of chronic hepatitis B. Hepatology 2016; 63(1):261-283.

3. Liaw YF, Sung JJ, Chow WC, Farrell G, Lee CZ, Yuen H, et al. Lamivudine for patients with chronic hepatitis B and advanced liver disease. N Engl J Med.2004; 351(15):1521-1531.

4. Bravo AA, Sheth SG, Chopra S. Liver biopsy. N Engl J Med. 2001; 344(7):495-500.

5. Teshale E, Lu M, Rupp LB, Holmberg SD, Moorman AC, Spradling P, et al. APRI and FIB-4 are good predictors of the stage of liver fibrosis in chronic hepatitis B: the Chronic Hepatitis Cohort Study (CHeCS). J Viral Hepat. 2014; 21(12):917-920.

6. Xiao G, Yang J, Yan L. Comparison of diagnostic accuracy of aspartate aminotransferase to platelet ratio index and fibrosis-4 index for detecting liver fibrosis in adult patients with chronic hepatitis B virus infection: a systemic review and meta-analysis. Hepatology. 2015; 61(1):292-302.

7. Vallet-Pichard A, Mallet V, Nalpas B, Verkarre V, Nalpas A, Dhalluin-Venier $\mathrm{V}$, et al. FIB-4: an inexpensive and accurate marker of fibrosis in HCV infection. comparison with liver biopsy and fibrotest. Hepatology. 2007; 46(1):32-36.

8. Cheng J, Hou J, Ding H, Chen G, Xie Q, Wang Y, et al. Validation of Ten Noninvasive Diagnostic Models for Prediction of Liver Fibrosis in Patients with Chronic Hepatitis B. PLoS One. 2015; 10(12):e0144425.

9. Kim WR, Berg T, Asselah T, Flisiak R, Fung S, Gordon SC, et al. Evaluation of APRI and FIB-4 scoring systems for non-invasive assessment of hepatic fibrosis in chronic hepatitis B patients. J Hepatol. 2016; 64(4):773-780.

10. Lemoine M, Shimakawa Y, Nayagam S, Khalil M, Suso P, Lloyd J, et al. The gamma-glutamyl transpeptidase to platelet ratio (GPR) predicts significant liver fibrosis and cirrhosis in patients with chronic HBV infection in West Africa. Gut. 2016; 65(8):1369-1376.

11. Li Q, Li W, Huang Y, Chen L. The gamma-glutamyl transpeptidase-to-platelet ratio predicts liver fibrosis and cirrhosis in $\mathrm{HBeAg}$-positive chronic $\mathrm{HBV}$ infection patients with high HBV DNA and normal or mildly elevated alanine transaminase levels in China. J Viral Hepat. 2016; 23(11):912-919.

12. Zhang W, Sun M, Chen G, An Y, Lv C, Wang Y, et al. Reassessment of gamma-glutamyl transpeptidase to platelet ratio (GPR): a large-sample, dynamic study based on liver biopsy in a Chinese population with chronic hepatitis B virus (HBV) infection. Gut. 2018; 67(5):989-991.

13. Coskun BD, Altinkaya E, Sevinc E, Ozen M, Karaman H, Karaman A, et al. The diagnostic value of a globulin/platelet model for evaluating liver fibrosis in chronic hepatitis B patients. Rev Esp Enferm Dig. 2015; 107(12):740-744.

14. $\mathrm{Li} \mathrm{O}, \mathrm{Lu} \mathrm{C}, \mathrm{Li} \mathrm{W}$, Huang $\mathrm{Y}$, Chen L. Globulin-platelet model predicts significant fibrosis and cirrhosis in CHB patients with high HBV DNA and mildly elevated alanine transaminase levels. Clin Exp Med. 2018; 18(1):71-78.

15. Kim HJ, Lee HK, Cho JH. Factor analysis of the biochemical markers related to liver cirrhosis. Pak J Med Sci. 2015; 31(5):1043-1046.

16. Takaki S, Kawakami Y, Miyaki D, Nakahara T, Naeshiro N, Murakami E, et al. Non-invasive liver fibrosis score calculated by combination of virtual touch tissue quantification and serum liver functional tests in chronic hepatitis C patients. Hepatol Res. 2014; 44(3):280-287.

17. Farid K, Omran MM, Farag RE, Arafa MM, Emran TM. Development and evaluation of a novel score for prediction of large oesophageal varices in patients with hepatitis c virus-induced liver cirrhosis. Br J Biomed Sci. 2017; 74(3):138-143.

18. Sarin SK, Kumar M, Lau GK, Abbas Z, Chan HL, Chen CJ, et al. Asian-Pacific clinical practice guidelines on the management of hepatitis B: a 2015 update. Hepatol Int. 2016; 10(1):1-98.

19. Bedossa P, Poynard T. An algorithm for the grading of activity in chronic hepatitis C. The METAVIR Cooperative Study Group. Hepatology. 1996; 24(2):289-293.

20. Shiha G, Ibrahim A, Helmy A, Sarin SK, Omata M, Kumar A, et al. Asian-Pacific Association for the Study of the Liver (APASL) consensus 
guidelines on invasive and non-invasive assessment of hepatic fibrosis: a 2016 update. Hepatol Int. 2017; 11(1):1-30.

21. Mehta SH, Lau B, Afdhal NH, Thomas DL. Exceeding the limits of liver histology markers. J Hepatol. 2009; 50(1):36-41.

22. Jiang W, Huang S, Teng H, Wang P, Wu M, Zhou X, et al. Diagnostic accuracy of point shear wave elastography and transient elastography for staging hepatic fibrosis in patients with non-alcoholic fatty liver disease: a meta-analysis. BMJ Open. 2018; 8(8):e021787.

23. Iijima H, Tada T, Kumada T, Kobayashi N, Yoshida M, Aoki T, et al. Comparison of liver stiffness assessment by transient elastography and shear wave elastography using six ultrasound devices. Hepatol Res. 2019; 49(6):676-686.

24. Lefebvre T, Wartelle-Bladou C, Wong P, Sebastiani G, Giard JM, Castel H, et al. Prospective comparison of transient, point shear wave, and magnetic resonance elastography for staging liver fibrosis. Eur Radiol. 2019; 29(12):6477-6488.

25. Wai CT, Greenson JK, Fontana RJ, Kalbfleisch JD, Marrero JA, Conjeevaram $\mathrm{HS}$, et al. A simple noninvasive index can predict both significant fibrosis and cirrhosis in patients with chronic hepatitis C. Hepatology. 2003; 38(2):518-526.

26. Vallet-Pichard A, Mallet V, Pol S. FIB-4: a simple, inexpensive and accurate marker of fibrosis in HCV-infected patients. Hepatology. 2006; 44(3):769-770.

27. Castera L. Noninvasive methods to assess liver disease in patients with hepatitis B or C. Gastroenterology. 2012; 142(6):1293-1302 e1294.

28. Estrabaud E, Vidaud M, Marcellin P, Asselah T. Genomics and HCV infection: progression of fibrosis and treatment response. J Hepatol. 2012; 57(5):1110-1125.

29. Bashour FN, Teran JC, Mullen KD. Prevalence of peripheral blood cytopenias (hypersplenism) in patients with nonalcoholic chronic liver disease. Am J Gastroenterol. 2000; 95(10):2936-2939.

30. Wright M, Goldin R, Fabre A, Lloyd J, Thomas H, Trepo C, et al. Measurement and determinants of the natural history of liver fibrosis in hepatitis $C$ virus infection: a cross sectional and longitudinal study. Gut. 2003; 52(4):574-579.

31. Liang XE, Dai L, Yang SL, Zhong CX, Peng J, Zhu YF, et al. Combining routine markers improves the accuracy of transient elastography for hepatitis B cirrhosis detection. Dig Liver Dis. 2016; 48(5):512-518.

32. Kayadibi H, Yasar B, Ozkara S, Serdar MA, Kurdas OO, Gonen C. The diagnostic accuracy of the Forns index, platelet count and AST to Platelet Ratio Index derived fibrosis index for the prediction of Hepatitis $\mathrm{C}$ virus-related significant liver fibrosis and cirrhosis. Scand J Clin Lab Invest. 2014; 74(3):240-247.

33. Peck-Radosavljevic M, Zacherl J, Meng YG, Pidlich J, Lipinski E, Langle F, et al. Is inadequate thrombopoietin production a major cause of thrombocytopenia in cirrhosis of the liver? J Hepatol. 1997; 27(1):127-131.

34. Adinolfi LE, Giordano MG, Andreana A, Tripodi MF, Utili R, Cesaro G, et al. Hepatic fibrosis plays a central role in the pathogenesis of thrombocytopenia in patients with chronic viral hepatitis. Br J Haematol. 2001; 113(3):590-595.

35. Lin ZH, Xin YN, Dong QJ, Wang Q, Jiang XJ, Zhan SH, et al. Performance of the aspartate aminotransferase-to-platelet ratio index for the staging of hepatitis C-related fibrosis: an updated meta-analysis. Hepatology. 2011; 53(3):726-736.

36. Holmberg SD, Lu M, Rupp LB, Lamerato LE, Moorman AC, Vijayadeva V, et al. Noninvasive serum fibrosis markers for screening and staging chronic hepatitis C virus patients in a large US cohort. Clin Infect Dis. 2013; 57(2):240-246.

37. Lin $\mathrm{CL}$, Liu $\mathrm{CH}$, Wang CC, Liang CC, Su TH, Liu CJ, et al. Serum Biomarkers Predictive of Significant Fibrosis and Cirrhosis in Chronic Hepatitis B. J Clin Gastroenterol. 2015; 49(8):705-713.

38. Jia J, Hou J, Ding H, Chen G, Xie $\mathrm{Q}$, Wang $\mathrm{Y}$, et al. Transient elastography compared to serum markers to predict liver fibrosis in a cohort of Chinese patients with chronic hepatitis B. J Gastroenterol Hepatol. 2015; 30(4):756-762.

39. Lesmana CR, Salim S, Hasan I, Sulaiman AS, Gani RA, Pakasi LS, et al. Diagnostic accuracy of transient elastography (FibroScan) versus the aspartate transaminase to platelet ratio index in assessing liver fibrosis in chronic hepatitis B: the role in primary care setting. J Clin Pathol. 2011; 64(10):916-920.

40. Zhu X, Wang LC, Chen EQ, Chen XB, Chen LY, Liu L, et al. Prospective evaluation of FibroScan for the diagnosis of hepatic fibrosis compared with liver biopsy/AST platelet ratio index and FIB-4 in patients with chronic HBV infection. Dig Dis Sci. 2011; 56(9):2742-2749.

41. Ren T, Wang H, Wu R, Niu J. Gamma-Glutamyl Transpeptidase-to-Platelet Ratio Predicts Significant Liver Fibrosis of Chronic Hepatitis B Patients in China. Gastroenterol Res Pract. 2017; 2017:7089702.

42. Wang RQ, Zhang OS, Zhao SX, Niu XM, Du JH, Du HJ, et al Gamma-glutamyl transpeptidase to platelet ratio index is a good noninvasive biomarker for predicting liver fibrosis in Chinese chronic hepatitis B patients. J Int Med Res. 2016; 44(6):1302-1313.

43. Wu D, Rao Q, Chen W, Ji F, Xie Z, Huang K, et al. Development and validation of a novel score for fibrosis staging in patients with chronic hepatitis B. Liver Int. 2018; 38(11):1930-1939.

44. Dong M, Wu J, Yu X, Li J, Yang S, Qi X, et al. Validation and comparison of seventeen noninvasive models for evaluating liver fibrosis in Chinese hepatitis B patients. Liver Int. 2018; 38(9):1562-1570.

45. Peng Y, Li Y, He Y, Wei $Q$, Xie $Q$, Zhang L, et al. The role of neutrophil to lymphocyte ratio for the assessment of liver fibrosis and cirrhosis: a systematic review. Expert Rev Gastroentero Hepatol. 2018; 12(5):503-513. 